\title{
Perspectives actuelles et futures de l'organisation et de la gestion de l'eau au Royaume Uni
}

\author{
Present and future perspectives for the organisation and management of \\ water resources in the United Kingdom
}

par John Seager

The Environment Agency, UK

(Traduction du texte original par Lucile Laffon, Agence de l'eau Adour-Garonne)

This paper briefly highlights some of the principal issues related to the organisation and management of water in the UK following the privatisation of the water services industry and the establishment of a new regulatory regime. Many lessons have been learnt since the re-organisation and the system is now becoming increasingly more efficient as experience is gained. The need to respond to new and predicted future pressures is stimulating new thinking on how the water environment can be managed in a more sustainable manner and what additional measures are needed. The paper describes the basic organisation of the water industry and how it is regulated; the investment planning process and the results have been achieved; the arrangements for river basin management; the state of the water environment and the principal issues to be tackled; and some of the challenges in managing the water environment in the context of sustainable development.

\section{I $\square$ ORGANISATION ET RÉGLEMENTA- TION DE L'INDUSTRIE DE L'EAU}

La structure organisationnelle au Royaume-Uni est compliquée par le fait que plusieurs dispositifs très différents les uns des autres existent en Angleterre et Pays de Galles, Ecosse et Nord-Irlande. L'accent politique actuellement mis sur la décentralisation pourrait conduire à de plus amples divergences. La principale différence est que les industries de service d'eau ont été privatisées en Angleterre et Pays de Galles en 1989, alors que cela n'a pas été fait en Ecosse et Nord-Irlande. Les différences majeures sont présentées dans le tableau 1, qui montre qu'en Angleterre et Pays de Galles, les services de distribution d'eau et d'assainissement sont effectués par des sociétés privées individuelles, devant rendre des comptes aux actionnaires, alors qu'en Ecosse et Nord-Irlande, ces services sont encore fournis par des autorités de l'eau appartenant au domaine public.

Bien que les dispositifs des services de l'industrie de l'eau soient différents, chaque pays a établi, via la loi sur l'Environnement de 1996, des institutions publiques* pour la protection de l'environnement et le contrôle de la pollution. Il s'agit respectivement de l'Agence de l'Environnement (Environment Agency, EA) pour l'Angleterre et le Pays de

* "regulatory bodies" ou "regulators" en anglais. Le terme signifie les organisations et les institutions chargées du développement des réglementations comme celles chargées de leur mise en cuvre et du contrôle de leur application.
Galles, de l'Agence Ecossaise de Protection de l'Environnement (Scottish Environmental Protection Agency, SEPA) et du Service Nord Irlandais de l'Environnement et du Patrimoine (Northern Ireland Environment and Heritage Service, NIEHS). De larges pouvoirs de régulation ont été donnés à ces organisations qui constituent une part clé de la stratégie gouvernementale de développement durable. Suite à la réorganisation importante de 1996, ces institutions publiques sont maintenant devenues complètement opérationnelles et auront une influence grandissante sur la gestion de l'eau et de l'environnement dans le futur.

En Angleterre et Pays de Galles, le régime de l'après privatisation a également créé d'autres institutions publiques importantes. La structure institutionnelle est résumée sur la figure 1. Le Bureau des Services de l'Eau (Office of Water Services, OFWAT) est responsable du contrôle financier des sociétés privées d'eau. Cela est effectué à travers le contrôle du prix de l'eau à travers un "Indice des prix de l'INSEE" (Retail price index, RPI) + un facteur K (positif ou négatif), établi pour les sociétés individuelles sur la base d'hypothèses d'amélioration de l'efficacité. C'est un point important car les sociétés d'eau opèrent en réalité dans une situation de monopole et les intérêts des particuliers doivent être protégés. OFWAT est également chargé, pour les sociétés d'eau, d'établir des normes sur les services, normes qui correspondent aux conditions de base auxquelles les sociétés doivent satisfaire pour obtenir leur autorisation. La définition d'objectifs de réduction des fuites au niveau des systèmes de 


\begin{tabular}{|c|c|c|c|}
\hline Services de l'eau & $\begin{array}{c}\text { Angleterre et Pays } \\
\text { de Galles }\end{array}$ & Ecosse & Nord-Irlande \\
\hline $\begin{array}{l}\text { Ressources en eau : planifi- } \\
\text { cation et autorisation }\end{array}$ & Agence de l'Environnement & $\begin{array}{l}\text { Secrétariat de l'autorité de } \\
\text { l'eau de l'Etat }\end{array}$ & $\begin{array}{l}\text { Département de l'Envi- } \\
\text { ronnement de Nord- } \\
\text { Irlande (DOE -NI) } \\
\text { Service de l'eau }\end{array}$ \\
\hline $\begin{array}{l}\text { Ressources en eau : déve- } \\
\text { loppement }\end{array}$ & $\begin{array}{l}\text { Sociétés d'eau et d'assai- } \\
\text { nissement } \\
\text { Sociétés de distribution } \\
\text { d'eau }\end{array}$ & Autorités de l'eau & $\begin{array}{l}\text { Département de l'Envi- } \\
\text { ronnement de Nord- } \\
\text { Irlande (DOE -NI) } \\
\text { Service de l'eau }\end{array}$ \\
\hline Distribution d'eau & $\begin{array}{l}\text { Sociétés d'eau et d'assai- } \\
\text { nissement } \\
\text { Sociétés de distribution } \\
\text { d'eau }\end{array}$ & Autorités de l'eau & $\begin{array}{l}\text { Département de l'Envi- } \\
\text { ronnement de Nord- } \\
\text { Irlande (DOE -NI) } \\
\text { Service de l'eau }\end{array}$ \\
\hline Assainissement & $\begin{array}{l}\text { Sociétés d'eau et d'assai- } \\
\text { nissement (i) }\end{array}$ & Autorités de l'eau & $\begin{array}{l}\text { Département de l'Envi- } \\
\text { ronnement de Nord- } \\
\text { Irlande (DOE -NI) } \\
\text { Service de l'eau }\end{array}$ \\
\hline $\begin{array}{l}\text { Protection de l'Environne- } \\
\text { ment et contrôle de la pol- } \\
\text { lution }\end{array}$ & Agence de l'Environnement & SEPA & $\begin{array}{l}\text { DOE (NI) } \\
\text { Service de l'Environne- } \\
\text { ment et du patrimoine }\end{array}$ \\
\hline $\begin{array}{l}\text { Lutte contre les inondations } \\
\text { et drainage des sols }\end{array}$ & $\begin{array}{l}\text { Autorités locales de } \\
\text { l'Agence de l'Environne- } \\
\text { ment }\end{array}$ & $\begin{array}{l}\text { Secrétariat de l'Etat et auto- } \\
\text { rités locales } \\
\text { Propriétaires riverains (drai- } \\
\text { nage des sols) }\end{array}$ & $\begin{array}{l}\text { Département de I'Agricul- } \\
\text { ture de Nord-Irlande }\end{array}$ \\
\hline
\end{tabular}

Tableau 1 - Responsabilités des services de l'eau au Royaume-Uni.

(i) La majorité des "Conseils généraux" (District Councils) et des "Conseils des grandes agglomérations" (Metropolitan Borough Councils) ont, au sein de leur territoire, passé des accords locaux avec la société de distribution d'eau et d'assainissement, pour effectuer la maintenance des réseaux d’assainissement.

Remarque : les fonctions détaillées peuvent ne pas exactement correspondre.

distribution d'eau, établi pour les sociétés individuelles, en est un exemple récent.

Le contrôle de la qualité de l'eau potable est sous la responsabilité d'une autre organisation : l'Inspection de l'Eau Potable (Drinking Water Inspectorate), chargée de l'application et de l'évaluation de la conformité avec les normes établies dans la directive communautaire "Eau potable".

En résumé, les principes de base du système actuel pour la gestion de l'eau en Angleterre et Pays de Galles sont :

- un cadre central fort comprenant des institutions publiques nationales ;

- une gestion intégrée par bassin versant ;

- l'approvisionnement des services d'eau par des sociétés privées avec une régulation économique et un suivi des performances ;

- la couverture des coûts des services ;

- des mécanismes statutaires pour impliquer le public dans la prise de décision.

\section{II $\square$ INVESTISSEMENT, PLANIFICATION ET GESTION DES OUVRAGES}

L'une des conséquences majeures de la privatisation et du système de régulation indépendante du prix de l'eau est que la formulation du prix doit être revue régulièrement pour prendre en compte des circonstances nouvelles et le financement de nouvelles contraintes résultant à la fois de législations nationales et européennes. Cela est effectué par le Directeur Général d'OFWAT dans un processus cyclique quinquennal qui est maintenant connu sous le nom de "revue périodique" (Periodic Review). L'information grâce à laquelle cette analyse est effectuée est fournie par les sociétés d'eau et fait l'objet d'audits indépendants. L'industrie de l'eau est maintenant impliquée dans la troisième phase de planification et de gestion des ouvrages, également appelée "processus AMP-3" (pour Asset Management Planning). Ce processus cyclique a engendré d'importants débats relatifs à la manière 


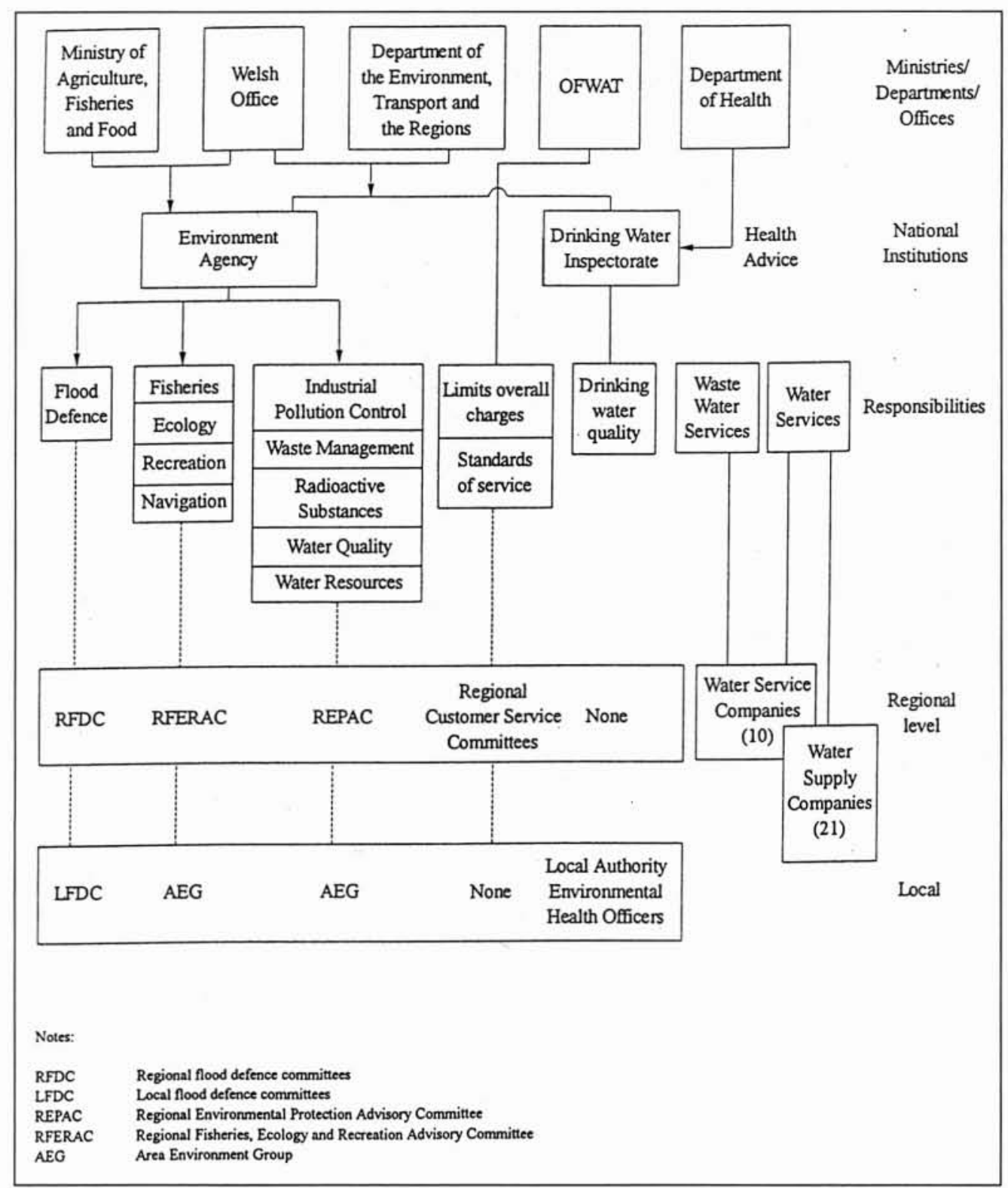

1. Résumé des relations institutionnelles principales pour la gestion de l'eau en Angleterre et au Pays de Galles.

de concilier les besoins de mise en concurrence de la part des consommateurs (valeur monétaire des taxes sur l'eau et cohérence avec les niveaux de service), les actionnaires (retour des investissements) et l'environnement (nécessité de satisfaire les normes environnementales de qualité et de protéger la totalité des écosystèmes). L'exercice de planification implique donc un dialogue et une négociation approfondis entre les services gouvernementaux et les institutions publiques chargées de la protection des divers intérêts, à savoir OFWAT et l'Inspection de l'Eau Potable (pour les consommateurs), les sociétés d'eau (pour les clients et actionnaires), l'Agence de l'Environnement (pour l'environnement). Cela a été un processus très instructif et beaucoup d'enseignements en ont été tirés. Malgré tout, cela a débouché sur des résultats très positifs et a focalisé les esprits sur le coût réel des services de l'eau et de l'amélioration de l'environnement ainsi que sur la volonté des consommateurs d'en payer le prix.

Depuis la privatisation, les dépenses des sociétés d'eau nécessaires à l'atteinte des niveaux de service exigés, des normes de qualité d'eau potable et de l'amélioration de l'environnement ont été substantielles. La figure 2 place dans son contexte historique l'évolution des investissements des industries de l'eau. Au cours des 5 années qui ont suivi la privatisation, les sociétés de service d'eau ont investi environ 14000 millions de Livres Sterling et le programme d'investissement jusqu'en 2005 est estimé à un volume supplémentaire de l'ordre de 24000 millions.

Cet investissement apporte aujourd'hui des améliorations significatives. Par exemple, en Angleterre et au Pays de Galles, la qualité des rivières s'est significativement améliorée de 1990 à 1995 avec plus de $25 \%$ de la longueur totale des rivières du pays pour lesquelles une amélioration nette de la qualité à la fois chimique et biologique est mise en évidence (figure 3). L'investissement relatif à l'assainissement et au traitement des eaux a été un facteur très important sous-jacent à cette évolution. Des améliorations supplémentaires sont attendues puisque les bénéfices des programmes d'investissement engagés vont porter leurs fruits. 


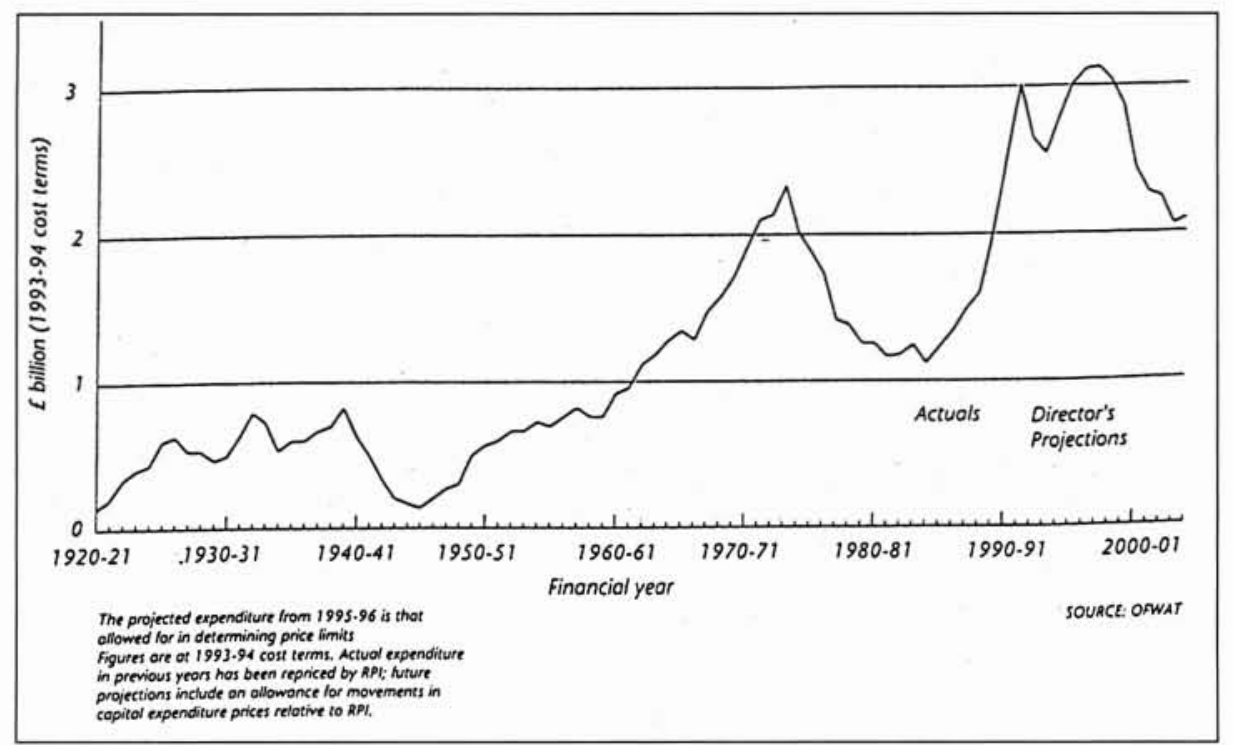

2. Dépenses d'investissement de l'industrie de l'eau.

\section{G GESTION DES BASSINS VERSANTS}

L'application de la gestion par bassin versant en tant que principe fondamental de la gestion de l'eau a un long passé au Royaume-Uni. La lutte contre les inondations, la gestion des ressources en eau, l'approvisionnement en eau potable, les services d'assainissement, la gestion des pêches et la protection des habitats des zones humides ont été organisés selon les principes de planification de la gestion des bassins depuis plusieurs années. Les structures de base des services d'eau et des institutions publiques reflètent les limites des principaux bassins hydrographiques.

Afin de développer le processus de planification des bassins versants et de l'intégrer avec les autres domaines de protection environnementale (tel que le contrôle des pollutions industrielles et les décharges), l'Agence de l'Environnement met en œuvre actuellement un programme de "Plans des Agences Locales de l'Environnement” (LEAPS) qui couvre l'ensemble des bassins versants d'Angleterre et du Pays de Galles. En 1999, lorsque le programme sera achevé, il y aura 133 LEAPS établissant des objectifs environnementaux locaux basés sur la réalité de terrain et comprenant un ensemble d'actions agréées impliquant les partenaires locaux.
Chaque plan aboutit grâce à un processus élargi de consultation impliquant l'ensemble des secteurs et des groupes intéressés et est à la disposition du public en général.

Chaque LEAP fournit un cadre de travail permettant :

- I'établissement d'objectifs locaux pour l'amélioration de la qualité de l'environnement ;

- la transposition des réglementations et priorités nationales en actions locales :

- l'évaluation des coûts et bénéfices des actions proposées ;

- les partenariats permettant de résoudre les problèmes environnementaux ;

- la promotion de la sensibilisation sur l'environnement à travers la formation et des interfaces avec les autorités chargées de la planification.

\section{IV — ÉTAT DE L'ENVIRONNEMENT ET PROBLÈMES À ABORDER}

L'Agence a récemment effectué un rapport sur l'état de l'environnement des eaux douces en Angleterre et au Pays de Galles [1]. Cela a conduit au développement d'un nouveau cadre de travail consistant en différents "points de vue" sur

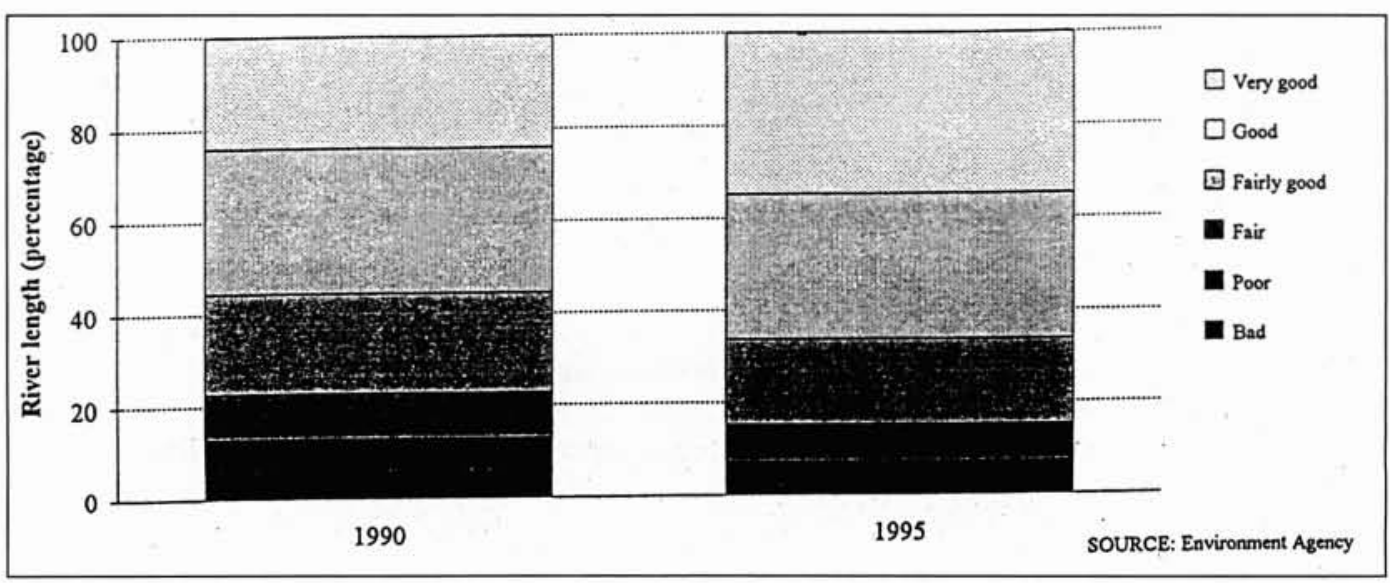

3. La qualité biologique des rivières en Angleterre et au Pays de Galles en 1990 et 1995. 
l'état de l'environnement [2] que l'Agence applique aujourd'hui dans ses évaluations environnementales. Les résultats disponibles montrent que quelques progrès très significatifs sont en cours dans les rivières de l'ensemble du pays. En plus des améliorations générales de la qualité des rivières mentionnées précédemment, des réductions significatives des rejets de substances toxiques ont été obtenues, résultat d'une réglementation plus contraignante, de changements dans les structures du monde industriel et agricole et de l'arrêt de l'utilisation de certains produits chimiques. Le nombre d'accidents de pollution répertoriés se réduit, ce qui reflète les efforts qui ont été mis en cuvre dans les campagnes de prévention de pollution. Des populations de poissons, de loutres et de quelques espèces d'oiseaux réapparaissent dans des régions où elles étaient absentes depuis plusieurs années.

Cependant, des problèmes importants restent à résoudre si I'on veut atteindre un équilibre plus durable entre les besoins de la société et la santé de l'environnement. Quelques-uns des problèmes prioritaires sont présentés ici :

- La modification du climat a des conséquences majeures dans la gestion de l'eau au Royaume-Uni compte-tenu de l'augmentation prévue du risque d'inondation, particulièrement dans la partie Est à basse altitude du pays, et des pressions sur les ressources en eau à la fois superficielles et souterraines de façon particulièrement aiguës dans le Sud et dans l'Est.

- Les prévisions relatives à la consommation des ménages et au changement de style de vie indiquent une augmentation des pressions sur les ressources en eau, à la fois en termes de qualité et de quantité, de plus en plus fortes. Les exigences prédites de 4,4 millions de foyers pour l'année 2020 vont créer des demandes nouvelles significatives pour l'approvisionnement en eau potable et la collecte des effluents. Il sera nécessaire de renverser les tendances récentes d'accroissement de la demande domestique en eau grâce à des programmes permettant de promouvoir la formation et la sensibilisation.

- La disparition et la modification des habitats ont été significatives dans l'environnement de l'Angleterre et du Pays de Galles, avec la disparition concomitante d'espèces floristiques et faunistiques qui dépendent des habitats hydrologiques. Récemment, on a constaté un déclin dans des régions de zones humides et dans des habitats et des étangs en bordures de cours d'eau. La restauration des habitats et la protection des espèces vulnérables est en cours dans le cadre du "Plan d'Action de la Biodiversité" au Royaume-Uni [3], dans lequel, parmi les actions prioritaires, sont identifiés les écosystèmes et les espèces aquatiques.

- Les sources de pollution diffuse des eaux souterraines et de surface restent un sujet inquiétant compte-tenu, notamment, des progrès significatifs réalisés dans le domaine de la réduction des pollutions ponctuelles. La gestion des pollutions diffuses requiert une approche très différente des méthodes traditionnelles de " bouts de tuyau ". Les outils d'évaluation des risques à l'échelle des bassins, qui s'appuient sur la technologie des Systèmes d'Information Géographiques, fournissent désormais des moyens très utiles pour cibler les efforts et les ressources dans les initiatives de prévention de la pollution. La formation est fondamentale pour améliorer la sensibilisation aux problèmes d'environnement et changer les comportements. De récentes campagnes de prévention de la pollution ont montré que des résultats positifs peuvent être atteints.

- L'augmentation rapide du nombre de produits chimiques mis sur le marché et utilisés est un problème majeur au regard du bien-être des hommes et de la nature. Les conséquences de l'exposition à des mélanges chimiques complexes ne sont pas bien comprises et il y a une inquiétude grandissante vis-à-vis des effets à long terme tels que les perturbations endocriniennes et les impacts sur la reproduction. Une réévaluation fondamentale des mécanismes permettant de prendre en compte les produits chimiques dans l'environnement est nécessaire en utilisant une approche basée sur les risques et en intégrant des contrôles des produits et des marchés ainsi que des programmes d'autorisation basés sur la toxicité et la surveillance de la qualité biologique.

- La qualité esthétique de l'environnement aquatique devient de plus en plus importante du fait que les activités récréatives aquatiques sont de plus en plus populaires. Beaucoup de personnes reviennent habiter dans des régions au bord de l'eau autrefois négligées et faisant l'objet de schémas d'aménagement urbains. La qualité esthétique est d'importance majeure du point de vue de la perception du public encore souvent délaissée dans la gestion de l'eau qui a eu tendance à s'appuyer sur des approches scientifiques et d'ingénierie lourdes. Davantage d'attention doit être portée à ce que pensent et ressentent les gens concernant leur environnement local et à ce qui peut être fait par les collectivités pour développer le sentiment que l'environnement appartient à tous et améliorer la qualité de vie.

\section{D PERSPECTIVES}

La gestion durable des ressources en eau requiert de plus en plus que soient développées des approches intégrées qui prennent en compte les besoins sociaux, économiques et environnementaux. De nombreuses discussions ont eu lieu sur ce sujet et de nouvelles idées sont progressivement introduites.

Les principes du projet de directive cadre sur les ressources en eau [4] vont donner un nouvel élan à la gestion de l'eau à travers l'Europe en mettant l'accent sur une approche davantage orientée vers l'écologie et l'utilisation de plans de gestion à l'échelle des bassins hydrographiques. Le Royaume-Uni a déjà développé une expérience considérable dans l'établissement d'objectifs écologiques et dans la mise en œuvre de programmes de surveillance permettant d'évaluer les progrès atteints vis-à-vis de ces objectifs. L'Agence de l'Environnement a récemment travaillé en collaboration avec les Agences de l'Eau françaises dans ce domaine, à travers un projet commun de comparaison des schémas de classification et de surveillance écologique entre les deux pays (annexe 1).

Il $\mathrm{y}$ a eu de multiples discussions à propos de l'utilisation d'approches complémentaires à la réglementation et, en particulier, sur le rôle des instruments économiques. La discussion a été ré-ouverte par la consultation récente du gouvernement sur les instruments économiques relatifs à la pollution de l'eau [5], qui a exploré les possibilités de mécanismes incitatifs de taxes, d'autorisations commerciales et de l'utilisation de la taxation comme moyen de prévention de la 
pollution (telles que des taxes sur les ventes de pesticides et de fertilisants). Un document supplémentaire est attendu sur l'utilisation d'instruments économiques permettant de réguler la demande en eau et de gérer les ressources.

Enfin, la gestion durable de l'environnement ne peut être menée avec succès qu'en prenant une vue holistique et sur le long terme. L'environnement de l'eau est seulement l'un des compartiments d'un système interactif et multimédia et ne peut pas être traité de façon isolée. L'Agence de l'Environnement est maintenant en train de développer une telle approche de gestion intégrée à travers sa "Stratégie Environnementale" [6]. Cela implique différents cadres de travail afin de mesurer l'état de l'environnement dans les différents milieux, d'évaluer les pressions polluantes exercées sur l'environnement, d'apprécier les risques, les coûts, les bénéfices et autres valeurs sociales, par la hiérarchisation des problèmes et le développement de plans d'action, et par la mise en œuvre de ces actions via des équipes pluridisciplinaires.

\section{VI $\square$ CONCLUSION}

La dernière décennie a connu une réorganisation fondamentale de l'organisation et de la gestion de l'eau au Royaume-Uni. Cela a été une période d'adaptation et d'apprentissage rapide, mais, malgré cela, des investissements considérables ont été entrepris et des améliorations significatives en ont résulté. Il y a cependant des problèmes en suspens qui doivent être abordés et qui vont requérir des changements fondamentaux dans les attitudes et les comportements du public en général et qui ne pourront pas être atteints seuls par l'industrie de l'eau et les institutions/personnes chargées de la réglementation. Cela va demander de renforcer la formation et l'information pour améliorer la sensibilisation et promouvoir l'utilisation durable des ressources en eau.

\section{RÉFÉRENCES BIBLIOGRAPHIQUES}

[1] Environment Agency (1998) - The State of the Environment of England and Wales : Fresh Waters. Stationery Office, London.

[2] Environment Agency (1998) - Viewpoints on the Environment : Developing a National Environmental Monitoring and Assessment Framework. Available from Environment Agency, Bristol.

[3] Department of the Environment (1994) - Biodiversity : the UK Action Plan. HMSO, London.

[4] European Commission (1998) - Commission Proposal for a Council Directive Establishing a Framework for Community Action in the Field of Water Policy. Official Journal No. C16, January 1998.

[5] Department of the Environment, Transport and the Regions (1997) Economic Instruments for Water Pollution Control. DETR, London.

[6] Environment Agency (1997) - An Environmental Strategy for the Millennium and Beyond. Available from Environment Agency, Bristol.

[7] Environment Agency, Agences de l'Eau, SNIFFER (July 1997) - Ecological quality of Fresh surface waters in Europe : research needs scoping study. Available from Environment Agency, Bristol ; Agences de I'Eau, France : SEPA, Edinburgh.

Annexe 1 : La collaboration franco-britannique dans le domaine de la qualité écologique des eaux de surface et le contexte de la Directive Cadre sur les ressources en eau

L'objectif de la collaboration franco-britannique est de contribuer au développement de la Directive Cadre sur les ressources en eau. Elle porte plus spécifiquement sur les méthodologies de surveillance et d'évaluation de la qualité écologique des cours d'eau.

Une première étude a été réalisée en 1997 et a fait l'objet d'une note de synthèse et d'un rapport commun aux six Agences de l'Eau françaises et aux Agences de l'Environnement d'Angleterre et du Pays de Galles, d'Ecosse et nordIrlande, présentés aux Etats Membres et à la Commission Européenne en juin 1997 [7].

L'étude comprend une analyse des pratiques actuelles de suivi et d'évaluation de la qualité chimique et écologique (expression de l'état chimique, biologique et hydromorphologique, tel que définie dans la Directive) mises en œuvre dans chaque pays au regard des exigences possibles de la Directive Cadre, en vue d'évaluer la faisabilité d'une harmonisation.

L'analyse met en évidence une grande convergence en ce qui concerne l'ensemble des objectifs relatifs à la gestion de l'eau et un accord sur les concepts majeurs tels que la nécessité de mettre en œuvre des dispositifs de surveillance et d'évaluation permettant à la fois d'apprécier l'état des eaux et de développer des programmes de restauration (sur la base des conditions chimiques, biologiques et physiques des cours d'eau). Cependant, des différences significatives au niveau des méthodes utilisées pour l'évaluation de la qualité existent et une harmonisation serait difficile et coûteuse.

Un second projet a donc été entrepris avec pour objectif d'étudier de manière plus approfondie la comparabilité des évaluations réalisées en France et Grande-Bretagne (à partir des spécifications techniques du projet de Directive et des méthodes nationales) et de proposer un mécanisme d'ajustement permettant des comparaisons directes et pouvant servir de modèle pour l'étude de la comparabilité au niveau européen.

Les résultats de cette seconde phase, attendus prochainement, seront disponibles auprès des Agences de l'Eau et des Agences de l'Environnement britanniques. 